\title{
Spatial and temporal coordination of insulin granule exocytosis in intact human pancreatic islets
}

\author{
Joana Almaça ${ }^{1,2,3}$ • Tao Liang ${ }^{4}$ - Herbert Y. Gaisano ${ }^{4}$ Hong Gil Nam ${ }^{3}$. \\ Per-Olof Berggren ${ }^{2,5,6,7}$ - Alejandro Caicedo ${ }^{1,8,9}$
}

Received: 29 May 2015 / Accepted: 12 August 2015 / Published online: 16 September 2015

(C) Springer-Verlag Berlin Heidelberg 2015

\begin{abstract}
Aims/hypothesis Insulin secretion is widely studied because it plays a central role in glucose homeostasis and diabetes. Processes from insulin granule fusion in beta cells to in vivo insulin secretion have been elucidated, but data at the cellular level do not fully account for several aspects of the macroscopic secretory pattern. Here we investigated how individual secretory events are coordinated spatially and temporally within intact human islets.

Methods We used the fluorescent probe neuropeptide $\mathrm{Y}$ (NPY)-pHluorin to visualise insulin granule secretion in isolated intact human islets.

Results We found that individual beta cells respond to increases in glucose concentration by releasing insulin granules in very discrete bursts with periods consistent with in vivo pulsatile insulin secretion. In successive secretory bursts during prolonged exposure to high glucose levels, secretory
\end{abstract}

events progressively localised to preferential release sites, coinciding with the transition to second phase insulin secretion. Granule secretion was very synchronised in neighbouring beta cells, forming discrete regional clusters of activity.

Conclusions/interpretation These results reveal how individual secretory events are coordinated to produce pulsatile insulin secretion from human islets.

Keywords Exocytosis · Human islets $\cdot$ Insulin granule Pulsatile secretion

$\begin{array}{ll}\text { Abbreviations } \\ \text { GFP } & \text { Green fluorescent protein } \\ \text { IBMX } & \text { 3-Isobutyl-1-methylxanthine } \\ \text { NPY } & \text { Neuropeptide Y }\end{array}$

Electronic supplementary material The online version of this article (doi:10.1007/s00125-015-3747-9) contains peer-reviewed but unedited supplementary material, which is available to authorised users.

Joana Almaça

jalmaca@med.miami.edu

$\triangle$ Per-Olof Berggren

Per-Olof.Berggren@ki.se

Alejandro Caicedo

acaicedo@med.miami.edu

1 Division of Endocrinology, Diabetes and Metabolism, Department of Medicine, University of Miami Miller School of Medicine, 1580 NW 10th Ave, Miami, FL 33136, USA

2 Diabetes Research Institute, University of Miami Miller School of Medicine, 1450 NW 10th Ave, Miami, FL 33136, USA
3 Center for Plant Aging Research, Institute for Basic Science and Department of New Biology, DGIST, Daegu, Republic of Korea Canada

5 Rolf Luft Research Center for Diabetes \& Endocrinology, Karolinska Institutet, Stockholm, Sweden

6 Lee Kong Chien School of Medicine, Nanyang Technical University, Singapore, Singapore

7 Imperial College, London, UK

8 Department of Physiology and Biophysics, Miller School of Medicine, University of Miami, Miami, FL, USA

9 Program in Neuroscience, Miller School of Medicine, University of Miami, Miami, FL, USA
4 Department of Medicine, University of Toronto, Toronto, ON, 
ROI Regions of interest

SNARE Soluble $N$-ethylmaleimide-sensitive factor attachment protein receptor

TIRF Total internal reflection fluorescence

\section{Introduction}

The beta cell of the pancreatic islet is the only cell in the body that secretes insulin, which in turn is the only hormone able to lower plasma glucose levels. Death or dysfunction of beta cells thus disturbs glucose homeostasis and leads to diabetes. Not surprisingly, beta cell function has been studied so exhaustively that the signalling pathways coupling glucose detection to insulin granule release are part of the physiological canon. Different aspects of insulin secretion, such as its doseresponse relationship for glucose and its dynamic temporal profile, have been examined in vitro and in vivo in many species including humans. A major feature of insulin secretion is its pulsatile pattern, in which most insulin is released in discrete bursts with periods ranging from 4 to $10 \mathrm{~min}[1,2]$. At the molecular level, insulin is packed in granules that are released in a $\mathrm{Ca}^{2+}$-dependent process similar to that in neurons, which is $\mathrm{Ca}^{2+}$-dependent and requires soluble $\mathrm{N}$-ethylmaleimide-sensitive factor attachment protein receptor (SNARE) proteins [3].

Granule exocytosis in beta cells has been studied using electrophysiological approaches, such as membrane capacitance measurements and amperometry [4-6], and microscopic approaches such as two-photon or total internal reflection fluorescence (TIRF) microscopy in combination with fluorescent probes and vesicle cargo proteins [7-11]. Data have mainly been obtained from clonal beta cell lines or dispersed beta cells, which do not allow the study of how insulin release is coordinated in the islet. Attempts at measuring granule exocytosis in beta cells within intact islets have been made only with rodent islets $[9,12,13]$, which differ structurally and functionally from human islets $[14,15]$.

For these reasons, it is still unclear how individual granule secretory events are coordinated in individual beta cells within an islet to produce a concerted secretory response that is pulsatile. Indeed, most studies show that insulin granules are released at a steady rate. Only two studies report a pulsatile pattern of granule fusion, though at a frequency not related to that of in vivo secretion $[11,13]$. We therefore investigated insulin granule exocytosis by using the fluorescent reporter neuropeptide Y (NPY)-pHluorin and confocal microscopy to visualise granule secretion in real time in beta cells within intact human islets. This technique allowed us to study the spatial and temporal patterns of granule secretion in individual beta cells as well as in beta cell populations throughout the human islet.

\section{Methods}

Islets We obtained human pancreatic islets from the Integrated Islet Distribution Program (NIDDK, NIH (Bethesda, MD, USA)). We received 11 healthy human islet preparations for this study but we observed responses to $\mathrm{KCl}$ and high glucose in only seven islet preparations that we used for the experiments included in the manuscript (male and female donors, $44.6 \pm 6.2$ years old, BMI $26.0 \pm 3.1 \mathrm{~kg} / \mathrm{m}^{2}$ ). Experiments have been approved by the ethics committee of the University of Miami.

Adenoviral production and islet infection Human islets were infected with adenoviruses containing NPY fused to the $\mathrm{pH}$-dependent green fluorescent protein (GFP) pHluorin for $48 \mathrm{~h}$ and cultured at $37^{\circ} \mathrm{C}$ for 5-7 days in CMRL 1066 (Cellgro, Herndon, Virginia, USA), 10\% (vol./vol.) FBS and $2 \mathrm{mmol} / 1 \mathrm{~L}$-glutamine [16]. Approximately $20-40 \%$ of the islet cells were infected. Most of the infected cells showed no background fluorescence, with few granules visible at basal glucose concentration ( $3 \mathrm{mmol} / \mathrm{l})$ in the absence of stimulation. Some cells had increased background fluorescence in the cytoplasm but did not show secretory events upon stimulation and were therefore not included in the analyses.

Confocal imaging and data quantification Human islets were placed on a coverslip in an imaging chamber (Warner instruments, Hamden, CT, USA) for imaging on a Leica TCS SP5 upright laser-scanning confocal microscope (Leica Microsystems, Wetzlar, Germany). Islets were continuously perfused with extracellular solution (in mmol/l: $125 \mathrm{NaCl}$, $5.9 \mathrm{KCl}, 2.56 \mathrm{CaCl}_{2}, 1 \mathrm{MgCl}_{2}, 25$ HEPES, $0.1 \%$ BSA, $3 \mathrm{mmol} / \mathrm{l}$ glucose, $\mathrm{pH} 7.4,37^{\circ} \mathrm{C}$ ) and confocal images were acquired with LAS AF software (Leica Microsystems) using a $63 \times$ water immersion objective (HCX APO L $63 \times / 0.9 \mathrm{NA}$ ). We used a resonance scanner for fast image acquisition to produce time-lapse recordings spanning $50 \mu \mathrm{m}$ of the islet (z-step: $5 \mu \mathrm{m}$, stack of ten confocal images with a size of $512 \times 512$ pixels) at $1.5 \mathrm{~s}$ resolution (XYZT imaging). NPYpHluorin fluorescence was excited at $488 \mathrm{~nm}$ and emission detected at $535-550 \mathrm{~nm}$. Insulin granule exocytosis was stimulated by increasing glucose concentration from 3 to $16 \mathrm{mmol} / \mathrm{l}$, in the presence of cAMP-raising agents 3isobutyl-1-methylxanthine (IBMX) $(100 \mu \mathrm{mol} / \mathrm{l})$ and forskolin $(10 \mu \mathrm{mol} / 1)$ [5] or by $\mathrm{KCl}$ depolarisation (30 mmol/l). cAMP-raising agents increased the consistency of secretory responses but did not change temporal patterns of granule secretion so that secretory events appeared in discrete bursts synchronised with the average cell response (see below) and with similar burst periods (range 1.4-6.6 min with IBMX/forskolin vs $1.5-10$ min without). Intracellular $\mathrm{pH}$ was increased by using $50 \mathrm{mmol} / \mathrm{l} \mathrm{NH} \mathrm{N}_{4} \mathrm{Cl}$ (to replace $\mathrm{NaCl}$ on an equimolar basis) and the plasma membrane was labelled 
with di-8-ANEPP $\left(2 \mu \mathrm{mol} / \mathrm{l}\right.$ for $1 \mathrm{~h}$ at $37^{\circ} \mathrm{C}$; Invitrogen $)$, excited at $488 \mathrm{~nm}$ and detected at $620 \mathrm{~nm}$.

ImageJ (http://imagej.nih.gov/ij/) was used to determine changes in fluorescence intensity over time in regions of interest (ROI) placed around single secretory events in confocal planes (see electronic supplementary material [ESM] Methods for a complete description of imaging data analysis). To identify sites of preferential secretion, we plotted fluorescence intensity along cell membranes and determined the number of secretory events that reappeared at the same site in two secretory bursts (adaptation from the birthday problem). Temporal stacks of images acquired during $30 \mathrm{~s} \mathrm{(} \mathrm{20}$ images) were used to calculate the $\mathrm{CV}$ of the fluorescence signal in the cell [17], according to the formula: $\mathrm{CV}=\mathrm{SD} /$ mean. Higher $\mathrm{CV}$ values indicate higher concentration of fluorescence signals. Pearson's correlation coefficient was used to determine co-localisation of secretory events in $30 \mathrm{~s}$ temporal stacks from two bursts.

Perifusion experiments Insulin secretion from non-infected or NPY-pHluorin-infected islets was measured 5 days after infection with adenoviruses as described previously [18].

Immunohistochemistry Human islets infected for 5 days with NPY-pHluorin were fixed with 4\% (vol./vol.) paraformaldehyde for 30-60 min on a microscope slide and immunostained for insulin, glucagon or somatostatin and GFP (Invitrogen, Carlsbad, CA, USA) using protocols previously described [15]. To assess co-localisation of NPY-pHluorin with islet hormones, we used ImageJ co-localisation analysis plugin 'Intensity Correlation Analysis' and calculated Mander's overlap coefficients $(R) . R$ values over 0.6 denote co-localisation [19].

Statistical analyses We used Prism 5.0 software (GraphPad Software, version 5, San Diego, CA, USA) to perform oneway analysis of variance (ANOVA) followed by a Tukey's multiple comparison test and considered statistical significance when $p$ values were lower than 0.05. Bar graphs show means \pm SEM.

\section{Results}

NPY-pHluorin works as a reporter for insulin secretory events We infected human islets with adenoviruses containing NPY-pHluorin, a fluorescent probe in which the vesicle cargo NPY is fused to the $\mathrm{pH}$-sensitive GFP (pHluorin) (Fig. 1a) [20]. By performing confocal time-lapse imaging in three dimensions (XYZT imaging), we visualised single secretory events in beta cells within the intact human islet (Fig. 1bf). In response to stimulation with high glucose (16 mmol/l), granules containing NPY-pHluorin became a
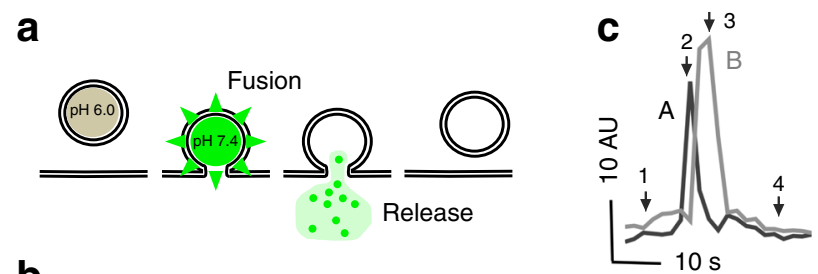

b

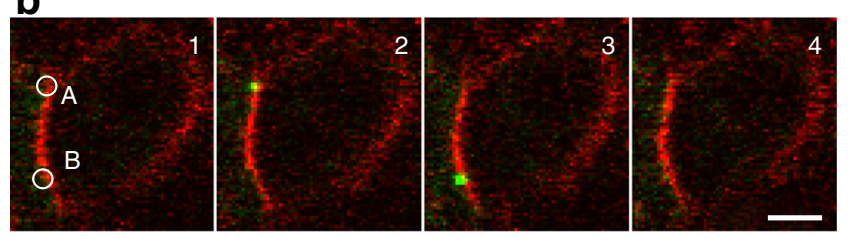

d

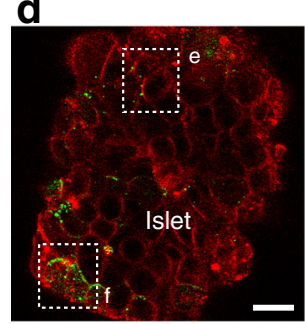

e

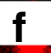

g

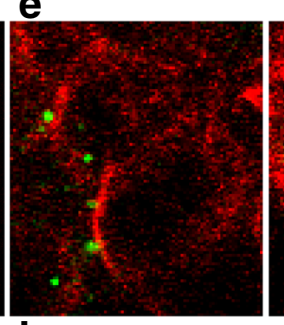

f

h
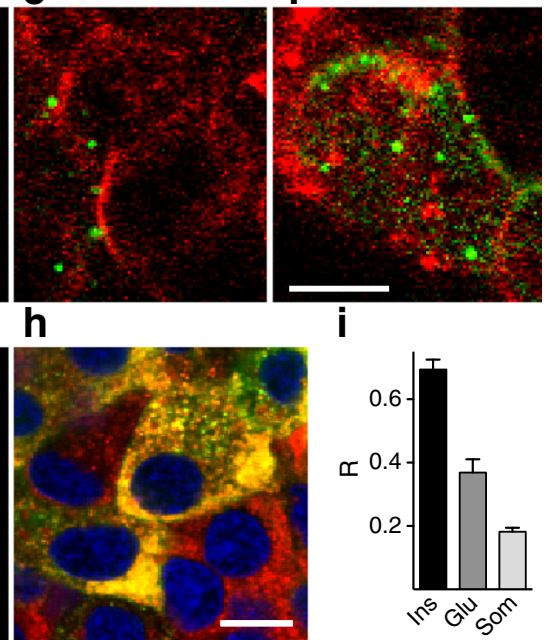

Fig. 1 Imaging insulin granule secretion in intact human islets. (a) The vesicle cargo NPY fused to pHluorin is used to follow insulin granule dynamics in real time. (b) Confocal images showing vesicle secretion (green) at the plasma membrane (red, labelled with di-8-ANEPP) of a cell within an intact human islet, triggered by high glucose, at particular times indicated in (c). (c) Traces of the changes in fluorescence intensity in ROIs A and B shown in (b); AU, arbitrary units. Arrows indicate time points of images in (b). (d-f) Temporal stack of 20 images (30 s) of a single confocal plane showing high glucose-triggered vesicle secretion in two cells (d) cut transversally by the confocal plane (e) and a cell cut tangentially and exposing its surface (f). (g, h) Confocal image (g) and higher magnification (h) of an isolated islet immunostained for insulin (red) and GFP (green). (i) Quantification of data in (g) showing Mander's overlap coefficients $(R), n=5$ islets. Ins, insulin; Glu, glucagon; Som, somatostatin. Scale bars, $5 \mu \mathrm{m}(\mathbf{b}, \mathbf{h}), 10 \mu \mathrm{m}(\mathbf{f})$ or $20 \mu \mathrm{m}(\mathbf{d}, \mathbf{g})$

visible upon fusing with the plasma membrane (Fig. 1b, $\mathrm{d}-\mathrm{f}$ ). These transient increases in NPY-pHluorin fluorescence could be readily recorded (Fig. 1c and ESM Video 1). $\mathrm{KCl}$ depolarisation also triggered an abrupt appearance of bright fluorescent structures at the cell surface (ESM Fig. 1). These secretory events were a minor fraction $(\sim 5 \%)$ of the total cellular pool of NPY-pHluorin-containing granules, as visualised by application of $\mathrm{NH}_{4} \mathrm{Cl}$ (ESM Fig. 1).

Most NPY-pHluorin-expressing cells were beta cells $(92 \pm$ $3 \%$ ) and only a small proportion of glucagon-containing alpha and somatostatin-containing delta cells were GFP-labelled (11 
$\pm 3 \%$ and $13 \pm 2 \%$ for alpha and delta cells, respectively, $n=5$ islets). Insulin co-localised with NPY-pHluorin, confirming that NPY fusion protein was stored in granules containing endogenous insulin (Fig. 1g-i). Although a subset of alpha cells was also infected with NPY-pHluorin $(\sim 10 \%)$, lowering the glucose concentration from 16 to $1 \mathrm{mmol} / \mathrm{l}$, a specific stimulus for alpha cells, did not elicit detectable granule fusion. Given their small number in the islet $(\sim 10 \%)$, the contribution of delta cells is negligible. While part of the exogenous NPY-pHluorin could segregate to granules other than those containing insulin [21], their glucose dependency and co-localisation with insulin indicate that most secretory events under high glucose conditions represent cargo release from insulin granules.

Although NPY has been shown to decrease insulin secretion from rodent islets $[22,23]$ and to be present in human islets [24], the total amount of glucose-stimulated insulin released from NPY-pHluorin-infected human islets was not different from that released from non-infected islets (AUC during 15 min stimulation with $16 \mathrm{mmol} / 1$ glucose was $0.14 \pm$ $0.01 \mathrm{ng} / \mu \mathrm{l} \times \min$ for non-infected islets and $0.11 \pm 0.005$ $\mathrm{ng} / \mu \mathrm{l} \times \min$ for NPY-pHluorin-infected islets, $p=0.11, n=3$ perifusions).

\section{Secretory events are highly synchronised and generate dis-} crete secretory bursts In healthy people insulin is secreted in pulses. Pulsatile secretion from the islet is crucial for the action of hormones in target tissues as it maintains a higher expression of insulin receptors at the membrane and facilitates post-insulin receptor signalling $[2,25]$. Insulin secretion oscillates in periods of $4-10 \mathrm{~min}$ in portal blood [26, 27]; a similar range has been observed in vitro with perfused pancreases or isolated islets from dogs $(10 \mathrm{~min})$, rhesus monkeys ( 6 $\mathrm{min}$ ) and humans (4-7 $\min$ [28-34]).

To determine how this regular pattern of insulin secretion is generated from multiple single secretory events, we stimulated NPY-pHluorin-infected islets with an elevated glucose concentration $(16 \mathrm{mmol} / \mathrm{l})$ for prolonged periods $(20 \mathrm{~min})$. Secretory events appeared in discrete bursts every $3.4 \pm$ 0.23 min (range 1.4-6.6 min; Fig. 2a, c and ESM Video 2). During each secretory burst, granules were very synchronised (Fig. 2b, d), with most granules ( $90 \%$ ) being secreted within a time difference of $4 \mathrm{~s}$ from the average cell response

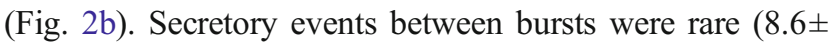
$1.0 \%, n=5$ cells, Fig. $2 \mathrm{~d}$ ). Our data show that individual secretory events are highly coordinated in beta cells within intact human islets.

Spatial pattern of granule secretion To ensure fast insulin release upon increases in glucose concentration, pools of insulin granules are pre-docked at the plasma membrane at specific sites (e.g. where syntaxin $1 \mathrm{~A} /$ munc 18 and other SNARE complexes are pre-assembled, or presynaptic structural
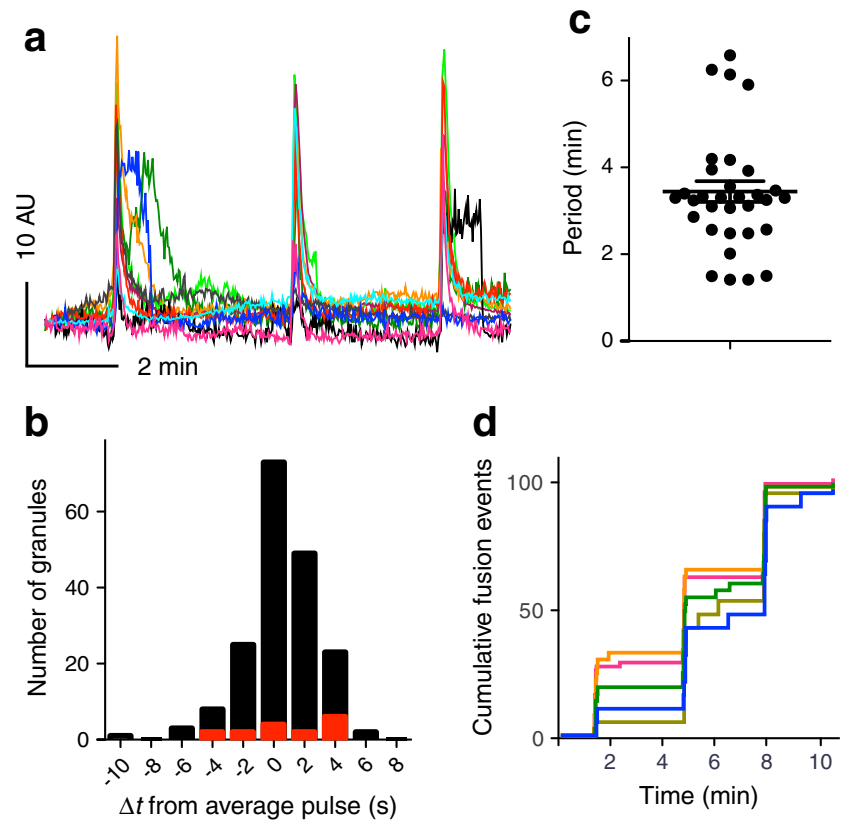

Fig. 2 Secretory events are highly synchronised and generate discrete pulses of secretion in individual beta cells. (a) Traces of fluorescence intensity in ROIs placed around individual secretory sites in a beta cell within an intact islet during sustained stimulation with $16 \mathrm{mmol} / \mathrm{l}$ glucose; AU, arbitrary units. Each colour represents an individual site. (b) Histogram showing the difference in time $(\Delta t)$ from the start of each secretory event to that of the cell response. Black bars, with IBMX/forskolin; red bars, without IBMX/forskolin. (c) Time between bursts of vesicle secretion (period), $n=9$ cells, $n=3$ islet preparations. Periods varied within a cell, from cell to cell, and from preparation to preparation. Line represents the average period \pm SEM. (d) Running sum of the number of secreted vesicles (each coloured trace represents secretory events from a single cell; $n=5$ cells, normalised to the total number of secretory events). Three bursts of vesicle secretion can be distinguished

proteins are enriched [35-37]). To determine whether granule secretion occurred randomly over the cell surface or preferentially at specific release sites, we compared the localisation of secretory events in successive secretory bursts. We found that some regions were used only once as release sites and others were used as exocytotic sites in two or three consecutive bursts (Fig. 3a, b). In the latter regions, several secretory events occurred in very close proximity, while the events that occurred in singly used release sites were usually isolated (Fig. 3b-d). To determine whether granule secretion preferentially occurred at specific membrane regions and not randomly over the cell surface, we used line plots of fluorescence intensity along the cell membrane (Fig. 3e) and calculated the probability of two secretory events in different bursts occurring at the same release site (see Methods and ESM Methods). We found that the proportion of secretory events reappearing in a subsequent burst was greater than that predicted to occur by chance (Fig. 3f), reflecting the contribution of repeated secretion at sites of preferred secretion. 

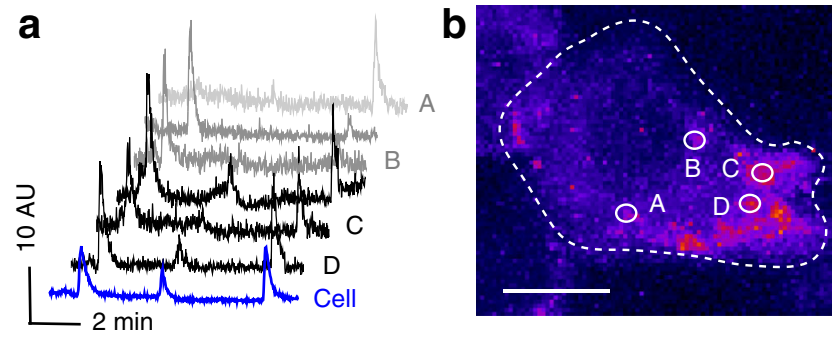

C
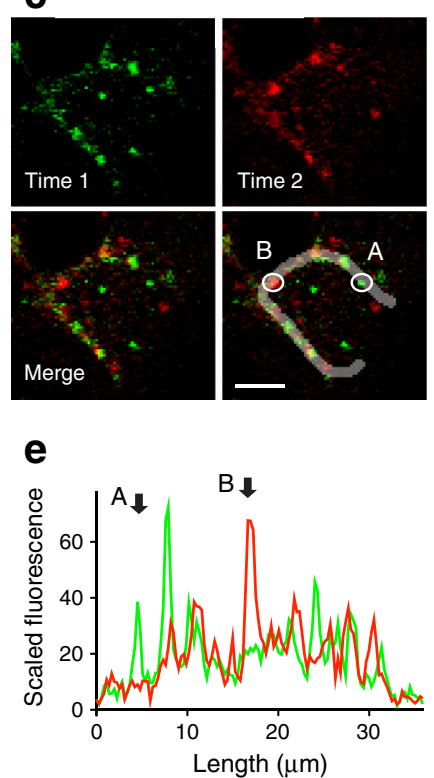

d
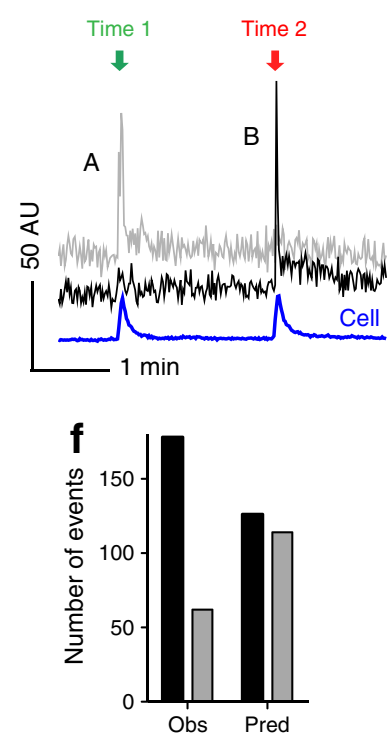

Fig. 3 Secretory events show distinct spatial patterns within a beta cell. (a) Traces of fluorescence intensity in ROIs placed around individual secretory sites during high glucose condition. At some sites secretion occurred repeatedly (black traces), while at others it only occurred once (grey traces); AU, arbitrary units. (b) Temporal stack (13 min $20 \mathrm{~s}$ ) of confocal images $(n=536)$ of a cell showing the sum of granule secretion during sustained stimulation with high glucose. Fluorescence intensity is highest where more secretory events occurred (pseudocolour scale). Circles denote ROIs for the traces shown in (a). (c) Temporal stacks ( $30 \mathrm{~s}$ ) of confocal images of a cell showing secretory events during two secretory bursts (first in green [Time 1], second in red [Time 2]). Scale bar, $5 \mu \mathrm{m}$ (b, c). (d) Traces of fluorescence intensity in ROIs placed around the individual secretory sites shown in (c). (e) Line scans along the cell membrane outline in (c) showing the profile of fluorescence intensity during the first (green) and second secretory burst (red). Secretory events did not recur at sites A and B (ROIs shown in [c]). (f) Quantification of recurrence of secretory events using the same line scans as those in (e). Bars represent values from a contingency table (black and grey bars for repeated and not repeated events, respectively). The observed number (Obs) of recurring secretory events (black bars) was higher than the predicted number (Pred) (Fisher exact test, $p<0.01, n=13$ cells, $n=4$ islets)

Release sites become more localised with each subsequent secretory burst Prolonged stimulation with high glucose triggered discrete secretory bursts in individual beta cells. We observed that in early bursts, granules were secreted in isolated and cluster-like release sites (Fig. 3), but with increasing burst number and duration of the high glucose stimulus, secretory sites progressively localised to sites of preferred secretion (Fig. 4a-d). To quantify this change in localisation of exocytotic sites, we calculated the $\mathrm{CV}$ of the fluorescence signals in temporal stacks of each burst. Greater $\mathrm{CV}$ values indicated that secretory events in later secretory bursts became

a

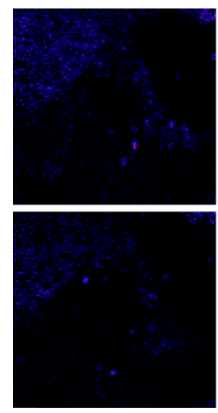

b

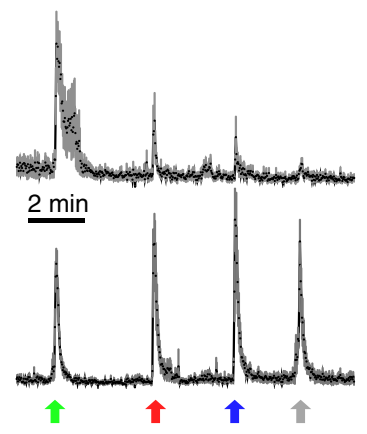

d

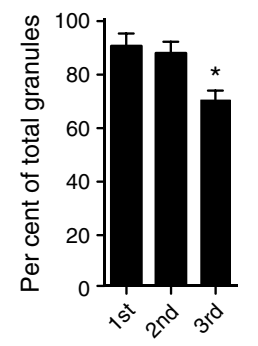

e

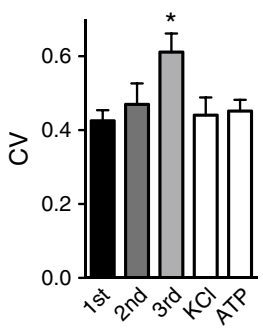

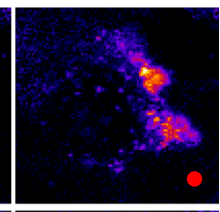

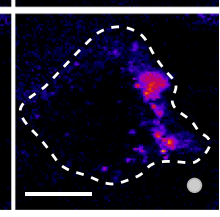

C

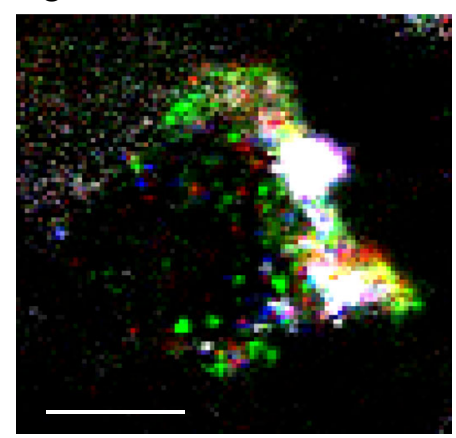

f

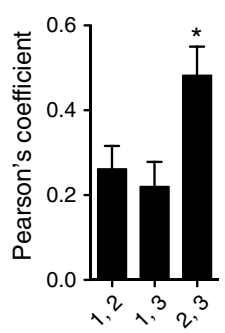

Fig. 4 Secretory events become more localised in subsequent bursts. (a) Sequence of eight temporal stacks $(20 \mathrm{~s})$ of confocal images of a cell within an intact islet showing the sum of secretory events before, between and during four secretory bursts (dots) in sustained $16 \mathrm{mmol} / 1$ glucose. Scale bar, $5 \mu \mathrm{m}$. (b) Average traces of fluorescence intensity in ROIs outside (top) and inside sites of preferential secretion (bottom) ( $n=7$ ROIs in cell shown in [a]). Peaks of top trace decrease, indicating fewer secretory events. Arrows indicate when images in (a) were taken. (c) Merged image of the temporal stacks of secretory bursts shown in (a). The sums of secretory events per burst are shown in different colours (colours as in $\mathbf{a}$ and $\mathbf{b}$ ). Sites of preferential secretion appear white. Scale bar, $5 \mu \mathrm{m}$. (d) The percentage of ROIs with a secretory event (based on recordings as in b) decreases with burst number, reflecting a reduction in secretory events outside regions of preferential secretion ( $n=7$ cells, ${ }^{*} p<0.05$ vs 1 st burst). (e) $\mathrm{CV}$ of cell fluorescence in secretory bursts (30 s temporal stacks) during high glucose, or upon $\mathrm{KCl}(30 \mathrm{mmol} / \mathrm{l})$ or ATP $(100 \mu \mathrm{mol} / \mathrm{l})$ stimulation $(n \geq 4$ cells, $* p<0.05$ for comparison between 1 st and 3rd bursts during high glucose). (f) Higher Pearson's correlation coefficient shows greater co-localisation of secretory events between pulses 2 and 3 than between pulses 1 and $2\left({ }^{*} p<0.05, n=5\right.$ cells $)$ 
more compartmentalised (Fig. 4e). In addition to a higher CV value, spatial co-localisation of secretory events between later bursts was also greater, as indicated by an increase in the Pearson coefficient (Fig. 4f). Stimulation with $\mathrm{KCl}$ after glucose or with ATP elicited granule secretion that was as broadly distributed as that of the first burst in high glucose, with $\mathrm{CV}$ values as low as those of the first burst (Fig. 4e). In conclusion, protracted stimulation with high glucose induced a progressive compartmentalisation of granule secretion that was specific to sustained glucose stimulation and was reversed after glucose concentration returned to basal levels.

\section{Secretory patterns reveal clusters of synchronous cells Be-} ta cells must respond in a coordinated way to generate a pulsatile pattern in insulin secretion from the islet. We therefore monitored secretion from multiple cells within the same islet and observed that secretory events in high glucose concentrations occurred within $10 \mathrm{~s}$ in beta cells localised in close proximity (Figs 2d, 5a-d and 6). Beta cells formed distinct regional clusters of synchronised activity. In contrast, beta cell clusters in different islet regions showed delays in their responses within a secretory burst ( $40 \mathrm{~s}$; Fig. 5). The sequence in the activation of the different clusters varied from burst to burst (Fig. 5c, d), suggesting that the response did not originate from a leading cluster. When $\mathrm{KCl}$ depolarisation was used as stimulus, the delay between clusters was absent and beta cells from different islet regions responded simultaneously (Fig. 5d, e).

The order in which beta cells showed exocytotic responses changed from burst to burst, indicating that there was no dominant cell serving as a pacemaker within a cluster (Fig. 6b).

\section{Discussion}

Our results show that imaging of NPY-pHluorin can be used to visualise insulin granule exocytosis in beta cells within intact human islets. We determined that in response to glucose stimulation insulin granules in individual beta cells are secreted in distinct bursts with exquisite synchronisation. These bursts appear in periods similar to those of pulsatile in vivo insulin secretion. With prolonged glucose stimulation and increasing pulse number, secretory events within a beta cell become localised to regions of preferred secretion. We further demonstrated that neighbouring human beta cells form distinct clusters comprised of $\sim 5-10$ cells in which activity is synchronised. These clusters of synchronised activity are preserved from burst to burst and differ from surrounding clusters in their timing within the global secretory pulse of the islet.

Most imaging studies on insulin exocytosis have been performed on either cell lines or isolated beta cells, mainly using TIRF microscopy (e.g. [8, 11, 35, 38-41]). Islet dissociation and cell culture induce cellular rearrangements that not only

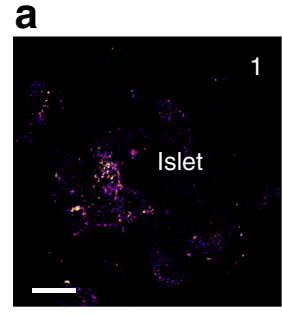

b

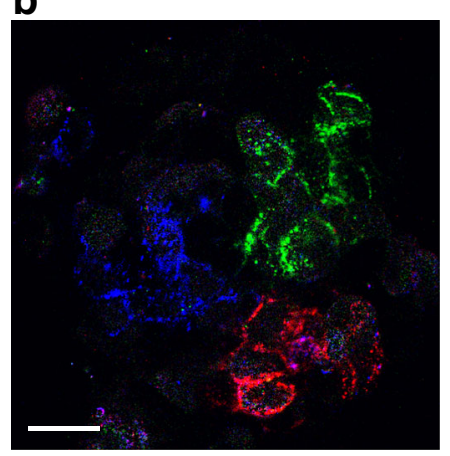

d

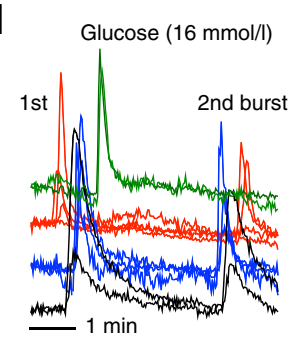

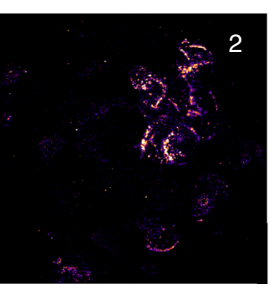

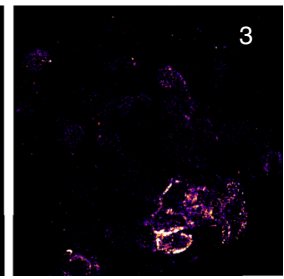

C

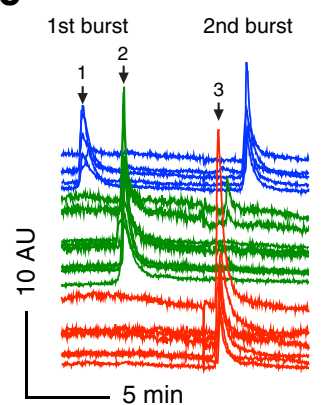

e
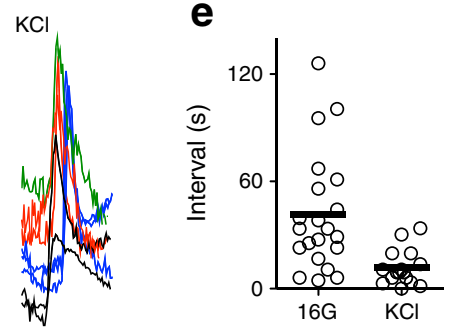

Fig. 5 Islet cells form discrete clusters of synchronised activity. (a) Sequence of temporal stacks (30 s) of confocal images showing secretory events stimulated by high glucose in an intact human islet. (b) Merged image of the temporal stacks shown in (a), with secretory events colourcoded: blue at time 1; green at time 2 and red at time 3 . Three nonoverlapping clusters comprising $\sim 10$ cells become visible. Scale bars, $20 \mu \mathrm{m}$. (c) Traces of secretory responses from cells shown in (b), with corresponding colours; AU, arbitrary units. Arrows indicate when images in (a) were acquired. (d) Traces of secretory responses from cells in response to high glucose and $\mathrm{KCl}$ stimulation (neighbouring cells in a cluster in same colour). The sequence in the activation of cell clusters changes from burst to burst. (e) Quantification of delays in the secretory responses between different clusters in the same islet stimulated with high glucose $(16 \mathrm{G})$ or $\mathrm{KCl}$. Lines represent average values $(n=3-6$ regions per islet, $n=3$ islets)

disrupt cell-to-cell communication, but may also affect granule pool distribution. Dispersed beta cells show marked variability in their responses to glucose due to metabolic heterogeneity and different activation thresholds. Because TIRF microscopy does not allow imaging of cells within intact islets, other imaging modalities have been used to measure insulin secretion in situ in the intact islet $[9,12,13,37]$. These experiments, however, were conducted on rodent islets, which are structurally different from human islets [14, 15]. Not surprisingly, our results with intact human islets have revealed features in insulin exocytosis not predicted by previous studies (see below). 
a

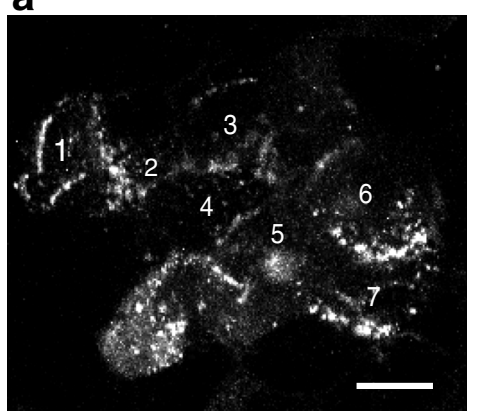

Fig. 6 The sequence of activation of cells within a cluster is not fixed. (a) Temporal stack of confocal images acquired during $30 \mathrm{~s}$ showing secretory events in a cell cluster within an intact islet. Numbers indicate different cells within the region. Scale bar, $10 \mu \mathrm{m}$. (b) Secretory responses of

Our findings show that multiple secretory events in individual beta cells occur almost simultaneously, within a narrow temporal window of $8 \mathrm{~s}$, and in discrete bursts. Few secretory events $(<9 \%)$ occurred in the periods between bursts. This pattern is in contrast to that found in other studies in which insulin granules underwent exocytosis at a steady rate, most likely as a result of averaging the behaviour of asynchronous dispersed cells. Our results are in line with those of a study on small clusters of human beta cells showing that insulin granules are secreted in recurrent discrete bursts [11]. However, bursts appeared with a periodicity of $15-45 \mathrm{~s}$, which is different from the periods we determined and from those of insulin secretion in vitro and in vivo [26, 27, 29, 31, 33, 34, 42].

Our findings further indicate that prolonged stimulation with high glucose changes the spatial pattern of granule secretion. With each subsequent secretory burst, granule secretion becomes more localised to preferred sites of exocytosis. We currently do not have a morphological or molecular correlate for the observed sites of preferred secretion, but they could have a high density of $\mathrm{P} / \mathrm{Q}$-type $\mathrm{Ca}^{2+}$ channels [43], be areas where SNARE proteins cluster [44] or be plasma membrane hubs for the delivery of components of the exocytotic machinery orientated towards the vasculature [36]. Interestingly, granule secretion becomes more confined at a time that coincides with the transition from first phase to the sustained second phase of insulin secretion ( $~ 5-10$ min into glucose stimulation) $[45,46]$. The transition to the second phase of insulin secretion can be explained by the depletion of primed granules and the recruitment of different functional pools of insulin granules with different release competence [3, 4]. Our results now suggest that this transition is also associated with changes in the spatial pattern of insulin granule secretion.

We also found that neighbouring beta cells form highly synchronised functional clusters of up to ten cells, consistent with the six to seven cells that are coupled through gap junctions in human islets [47]. There was no preferential sequence in which cells within a cluster were activated, which is consistent with the notion that electrical coupling lessens the b

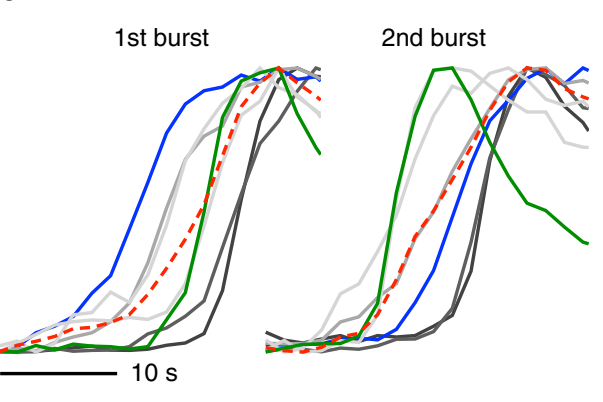

the cells shown in (a) in two distinct secretory bursts show different activation sequences (see blue and green traces). Red lines show the response of the whole region. Responses are scaled to peak fluorescence increase

effects of physiological cell-to-cell variations [48, 49]. In contrast to the fast coupling seen within clusters, longer time delays in activation and their spatial segregation within the islet suggest that coordination between clusters requires paracrine signalling. The looser coupling between cell clusters may explain why the overall secretory pulse of the islet is less discrete than that of granule secretion from individual cells or clusters [33, 34].

Characterising insulin exocytosis in vitro is a first step towards fully understanding the cellular mechanisms that orchestrate individual secretory events to achieve the complex oscillatory pattern of insulin release. The ability to measure insulin granule secretion in real time from multiple cells in intact human islets provides an exceptional tool that can be combined with mouse models of in vivo imaging of vascularised and innervated islets [50]. It is important to determine whether the subcellular spatial pattern of insulin granule secretion we report here is associated with vascular arrangements [36].

Funding This work was funded as follows: NIH grants R56DK084321 (AC) and R01DK084321 (AC); the Institute for Basic Science IBS-R013D1 (HGN); the Juvenile Diabetes Research Foundation (P-OB); the Swedish Research Council; the Novo Nordisk Foundation; the Swedish Diabetes Association; the Family Erling-Persson Foundation; the Skandia Insurance Company Ltd; Strategic Research Program in Diabetes at Karolinska Institutet; the Berth von Kantzow's Foundation; VIBRANT Grant FP7-2288933; the Knut and Alice Wallenberg Foundation and Lee Kong Chien School of Medicine, Nanyang Technical University, Singapore and Imperial College, London, UK ERC-2013-AdG 338936BetaImage. JA is a recipient of a postdoctoral fellowship from the American Heart Association (14POST20380499).

Duality of interest The authors declare that there is no duality of interest associated with this manuscript.

Contribution statement JA and AC conceived and designed the study and wrote the manuscript. P-OB, HGN and AC raised funding. JA acquired the data, JA and $\mathrm{AC}$ analysed the data and all authors interpreted the data. All authors revised the article and approved its final version. JA is the guarantor of this work. 


\section{References}

1. Tengholm A, Gylfe E (2009) Oscillatory control of insulin secretion. Mol Cell Endocrinol 297:58-72

2. Satin LS, Butler PC, Ha J, Sherman AS (2015) Pulsatile insulin secretion, impaired glucose tolerance and type 2 diabetes. Mol Asp Med 42:61-77

3. Gaisano HY (2014) Here come the newcomer granules, better late than never. Trends Endocrinol Metab 25:381-388

4. Rorsman P, Renstrom E (2003) Insulin granule dynamics in pancreatic beta cells. Diabetologia 46:1029-1045

5. Hanna ST, Pigeau GM, Galvanovskis J, Clark A, Rorsman P, MacDonald PE (2009) Kiss-and-run exocytosis and fusion pores of secretory vesicles in human beta-cells. Pflugers Arch 457:1343-1350

6. Barbosa RM, Silva AM, Tome AR, Stamford JA, Santos RM, Rosario LM (1998) Control of pulsatile 5-HT/insulin secretion from single mouse pancreatic islets by intracellular calcium dynamics. J Physiol 510:135-143

7. Bergeron A, Pucci L, Bezzi P, Regazzi R (2014) Analysis of synaptic-like microvesicle exocytosis of B cells using a live imaging technique. PLoS One 9:e87758

8. Ravier MA, Tsuboi T, Rutter GA (2008) Imaging a target of $\mathrm{Ca}^{2+}$ signalling: dense core granule exocytosis viewed by total internal reflection fluorescence microscopy. Methods 46:233-238

9. Takahashi N, Kishimoto T, Nemoto T, Kadowaki T, Kasai H (2002) Fusion pore dynamics and insulin granule exocytosis in the pancreatic islet. Science 297:1349-1352

10. Zhu D, Zhang Y, Lam PP et al (2012) Dual role of VAMP8 in regulating insulin exocytosis and islet beta cell growth. Cell Metab 16:238-249

11. Michael DJ, Xiong W, Geng X, Drain P, Chow RH (2007) Human insulin vesicle dynamics during pulsatile secretion. Diabetes 56:1277-1288

12. Li D, Chen S, Bellomo EA et al (2011) Imaging dynamic insulin release using a fluorescent zinc indicator for monitoring induced exocytotic release (ZIMIR). Proc Natl Acad Sci U S A 108:21063-21068

13. Low JT, Mitchell JM, Do OH et al (2013) Glucose principally regulates insulin secretion in mouse islets by controlling the numbers of granule fusion events per cell. Diabetologia 56:2629-2637

14. Brissova M, Fowler MJ, Nicholson WE et al (2005) Assessment of human pancreatic islet architecture and composition by laser scanning confocal microscopy. J Histochem Cytochem 53:1087-1097

15. Cabrera O, Berman DM, Kenyon NS, Ricordi C, Berggren PO, Caicedo A (2006) The unique cytoarchitecture of human pancreatic islets has implications for islet cell function. Proc Natl Acad Sci U S A 103:2334-2339

16. Zhu D, Koo E, Kwan E et al (2013) Syntaxin-3 regulates newcomer insulin granule exocytosis and compound fusion in pancreatic beta cells. Diabetologia 56:359-369

17. Hoppa MB, Collins S, Ramracheya R et al (2009) Chronic palmitate exposure inhibits insulin secretion by dissociation of $\mathrm{Ca}^{2+}$ channels from secretory granules. Cell Metab 10:455-465

18. Cabrera O, Jacques-Silva MC, Berman DM et al (2008) Automated, high-throughput assays for evaluation of human pancreatic islet function. Cell Transplant 16:1039-1048

19. Zinchuk V, Grossenbacher-Zinchuk O (2009) Recent advances in quantitative colocalization analysis: focus on neuroscience. Prog Histochem Cytochem 44:125-172

20. Miesenbock G, De Angelis DA, Rothman JE (1998) Visualizing secretion and synaptic transmission with $\mathrm{pH}$-sensitive green fluorescent proteins. Nature 394:192-195
21. Sobota JA, Ferraro F, Back N, Eipper BA, Mains RE (2006) Not all secretory granules are created equal: partitioning of soluble content proteins. Mol Biol Cell 17:5038-5052

22. Wang ZL, Bennet WM, Wang RM, Ghatei MA, Bloom SR (1994) Evidence of a paracrine role of neuropeptide- $Y$ in the regulation of insulin release from pancreatic islets of normal and dexamethasonetreated rats. Endocrinology 135:200-206

23. Morgan DG, Kulkarni RN, Hurley JD et al (1998) Inhibition of glucose stimulated insulin secretion by neuropeptide $\mathrm{Y}$ is mediated via the Y1 receptor and inhibition of adenylyl cyclase in RIN 5AH rat insulinoma cells. Diabetologia 41:1482-1491

24. Bennet WM, Wang ZL, Jones PM et al (1996) Presence of neuropeptide $\mathrm{Y}$ and its messenger ribonucleic acid in human islets: evidence for a possible paracrine role. J Clin Endocrinol Metab 81: $2117-2120$

25. Goodner CJ, Sweet IR, Harrison HC Jr (1988) Rapid reduction and return of surface insulin receptors after exposure to brief pulses of insulin in perifused rat hepatocytes. Diabetes 37:1316-1323

26. Porksen N, Grofte T, Greisen J et al (2002) Human insulin release processes measured by intraportal sampling. Am J Physiol Endocrinol Metab 282:E695-E702

27. Song SH, McIntyre SS, Shah H, Veldhuis JD, Hayes PC, Butler PC (2000) Direct measurement of pulsatile insulin secretion from the portal vein in human subjects. J Clin Endocrinol Metab 85:44914499

28. Bertram R, Sherman A, Satin LS (2010) Electrical bursting, calcium oscillations, and synchronization of pancreatic islets. Adv Exp Med Biol 654:261-279

29. Gilon P, Ravier MA, Jonas JC, Henquin JC (2002) Control mechanisms of the oscillations of insulin secretion in vitro and in vivo. Diabetes 51(Suppl 1):S144-S151

30. Goodner CJ, Koerker DJ, Stagner JI, Samols E (1991) In vitro pancreatic hormonal pulses are less regular and more frequent than in vivo. Am J Physiol 260:E422-E429

31. Hellman B, Salehi A, Gylfe E, Dansk H, Grapengiesser E (2009) Glucose generates coincident insulin and somatostatin pulses and antisynchronous glucagon pulses from human pancreatic islets. Endocrinology 150:5334-5340

32. Stagner JI, Samols E, Weir GC (1980) Sustained oscillations of insulin, glucagon, and somatostatin from the isolated canine pancreas during exposure to a constant glucose concentration. J Clin Invest 65:939-942

33. Ritzel RA, Veldhuis JD, Butler PC (2006) The mass, but not the frequency, of insulin secretory bursts in isolated human islets is entrained by oscillatory glucose exposure. Am J Physiol Endocrinol Metab 290:E750-E756

34. Ritzel RA, Veldhuis JD, Butler PC (2003) Glucose stimulates pulsatile insulin secretion from human pancreatic islets by increasing secretory burst mass: dose-response relationships. J Clin Endocrinol Metab 88:742-747

35. Gandasi NR, Barg S (2014) Contact-induced clustering of syntaxin and munc18 docks secretory granules at the exocytosis site. Nat Commun 5:3914

36. Low JT, Zavortink M, Mitchell JM et al (2014) Insulin secretion from beta cells in intact mouse islets is targeted towards the vasculature. Diabetologia 57:1655-1663

37. Takahashi N, Hatakeyama H, Okado H, Noguchi J, Ohno M, Kasai H (2010) SNARE conformational changes that prepare vesicles for exocytosis. Cell Metab 12:19-29

38. Shibasaki T, Takahashi H, Miki T et al (2007) Essential role of Epac2/Rap1 signaling in regulation of insulin granule dynamics by cAMP. Proc Natl Acad Sci U S A 104:19333-19338

39. Tsuboi T, Ravier MA, Parton LE, Rutter GA (2006) Sustained exposure to high glucose concentrations modifies glucose signaling and the mechanics of secretory vesicle fusion in primary rat pancreatic $\beta$-cells. Diabetes 55:1057-1065 
40. Uenishi E, Shibasaki T, Takahashi H et al (2013) Actin dynamics regulated by the balance of neuronal Wiskott-Aldrich syndrome protein (N-WASP) and cofilin activities determines the biphasic response of glucose-induced insulin secretion. J Biol Chem 288: 25851-25864

41. Krus U, King BC, Nagaraj V et al (2014) The complement inhibitor CD59 regulates insulin secretion by modulating exocytotic events. Cell Metab 19:883-890

42. Matveyenko AV, Liuwantara D, Gurlo T et al (2012) Pulsatile portal vein insulin delivery enhances hepatic insulin action and signaling. Diabetes 61:2269-2279

43. Rorsman P, Braun M (2013) Regulation of insulin secretion in human pancreatic islets. Annu Rev Physiol 75:155-179

44. Ohara-Imaizumi M, Fujiwara T, Nakamichi Y et al (2007) Imaging analysis reveals mechanistic differences between first- and secondphase insulin exocytosis. J Cell Biol 177:695-705

45. van Haeften TW, Pimenta W, Mitrakou A et al (2000) Relative contributions of beta-cell function and tissue insulin sensitivity to fasting and postglucose-load glycemia. Metab Clin Exp 49:1318-1325

46. Gembal M, Gilon P, Henquin JC (1992) Evidence that glucose can control insulin release independently from its action on ATP-sensitive $\mathrm{K}+$ channels in mouse B cells. J Clin Invest 89:1288-1295

47. Serre-Beinier V, Bosco D, Zulianello L et al (2009) Cx36 makes channels coupling human pancreatic $\beta$-cells, and correlates with insulin expression. Hum Mol Genet 18:428-439

48. Rocheleau JV, Remedi MS, Granada B et al (2006) Critical role of gap junction coupled KATP channel activity for regulated insulin secretion. PLoS Biol 4:e26

49. Speier S, Gjinovci A, Charollais A, Meda P, Rupnik M (2007) Cx36-mediated coupling reduces $\beta$-cell heterogeneity, confines the stimulating glucose concentration range, and affects insulin release kinetics. Diabetes 56:1078-1086

50. Speier S, Nyquist D, Cabrera O et al (2008) Noninvasive in vivo imaging of pancreatic islet cell biology. Nat Med 14:574-578 